\title{
Systematic calculation of finite-time mixed singular vectors and characterization of error growth for persistent coherent atmospheric disturbances over Eurasia
}

Courtney Quinn, ${ }^{1, \text { a) }}$ Terence J. O’Kane, ${ }^{1}$ and Dylan Harries ${ }^{1, b)}$

CSIRO Oceans and Atmosphere, Hobart, Tasmania, Australia

(Dated: 11 August 2021)

Singular vectors (SVs) have long been employed in the initialization of ensemble numerical weather prediction (NWP) in order to capture the structural organization and growth rates

of those perturbations or "errors" associated with initial condition errors and instability processes of the large scale flow. Due to their (super) exponential growth rates and spatial scales, initial SVs are typically combined empirically with evolved SVs in order to generate forecast perturbations whose structures and growth rates are tuned for specified lead-times. Here we present a systematic approach to generating finite time or "mixed" SVs (MSVs) based on a method for the calculation of covariant Lyapunov vectors (CLVs) and appropriate choices of the matrix cocycle. We first derive a data-driven reduced order model to characterize persistent geopotential height anomalies over Europe and Western Asia (Eurasia) over the period 1979-present from the NCEPv1 reanalysis. We then characterize and compare the MSVs and SVs of each persistent state over Eurasia for particular leadtimes from a day to over a week. Finally, we compare the spatio-temporal properties of SVs and MSVs in an examination of the dynamics of the 2010 Russian heatwave. We show that MSVs provide a systematic approach to generate initial forecast perturbations projected onto relevant expanding directions in phase space for typical NWP forecast lead-times.

This manuscript has been submitted for publication in Chaos. Please note that the manuscript is currently in peer-review. Subsequent versions of this manuscript may have slightly different content. If accepted, the final version of this manuscript will be available via the 'Peer-reviewed Publication DOI' link. Please feel free to contact the authors with any feedback.

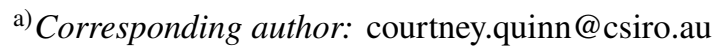

${ }^{b)}$ South Australian Health and Medical Research Institute
} 
Persistent atmospheric events, often coherent over thousands of kilometers, can have a large impact on daily weather conditions yet remain challenging to forecast. Most weather prediction centres now routinely use ensemble forecasts to estimate the range of uncertainties in weather forecasts of the near future. This involves initializing multiple forecasts in the directions of greatest instability in order to capture transitional behavior. Here, we first develop a reduced model for atmospheric regimes in the continental Europe-Asia sector of the Northern Hemisphere from which we explore different methods for identifying directions of unstable growth based on local (finite time) and global (asymptotic) dynamical vectors. We compute the local perturbation vectors optimized over an increasing number of days to compare their respective ability to project onto the synoptic time and spatial scales of interest typically associated with blocking. We then consider the specific case study of the 2010 Russian heat wave.

\section{INTRODUCTION}

When it comes to predicting atmospheric conditions, it has been long known that prediction of the distant future is practically impossible. Famously, Lorenz ${ }^{1}$ first showed that small errors in the initial conditions for even very simplified, but nonlinear, models of the atmosphere grow over finite time intervals before saturating, hence imposing a limit on the predictability of any future atmospheric state.

A particular challenge of weather forecasting involves estimating the uncertainty in the atmospheric basic state through predicting the probability density function of states in phase space. This is formally equivalent to estimating an infinite hierarchy of moments or cumulants in spectral space $^{2}$. Lorenz ${ }^{3}$ was the first to consider error growth by application of an ensemble generated as random initial errors added to a particular initial (analyzed) state. He showed that even simple reduced models for the atmosphere display varying leading error growth rates over consecutive 4day periods. Lorenz ${ }^{4}$ further showed that for the Northern Hemisphere synoptic scale troposphere, small errors in geopotential height states tended to double about every 8 days. In practice, forecast skill varies not simply due to errors in the initial conditions, i.e., the analyzed state after assimilation of observations, but also due to model biases in the representation of physical processes, finite grid resolution, and poor or even absent subgrid scale parameterizations. Most relevant to the present 
study, the variability of forecast skill is directly related to the instability of the large scale flow itself ${ }^{5}$.

The approach suggested by Lorenz relates to the linearization of the model in question where small errors evolve according to the system's tangent linear dynamics. The magnitude and directions of error growth can therefore be related to the singular values and singular vectors (SVs), respectively, of the tangent linear propagator (see Section II for mathematical details). Today, current methods for ensemble prediction attempt to directly sample rapidly expanding directions in phase space with appropriate initial perturbations whose spatio-temporal scales sample the region of phase space where the dynamics are relevant to the physical processes of interest. For example, the European Centre for Medium-Range Weather Forecasts (ECMWF) began exploring the behavior of SVs within ensemble forecasting in the $90 \mathrm{~s}^{6-11}$, leading to the SVs being formally integrated into the initial perturbations of the forecasting systems ${ }^{12}$. Leutbecher and Palmer ${ }^{13}$ provide a detailed description of the ECMWF ensemble prediction system whose perturbations are based on the leading part (vectors) of the singular value decomposition (SVD) of the tangent linear dynamics (linearized as a first order Taylor expansion for a particular solution of the nonlinear model) over a finite time interval.

While the approach using SVs as initial perturbations is optimal for disturbances that grow over the first day or two of the forecast, there is an increasing demand for medium-range forecasts which extend out to 10 days or longer. Such forecasts should reflect changes in the background flow, including large-scale persistent anomalies that develop and decay within that time frame. Beyond weather systems, for climate models whose respective domains extend over a large range of spatial and temporal scales, it is practically necessary to isolate the dynamical behavior on the scales of interest. One approach is through reduced models.

In Quinn, Harries, and O'Kane ${ }^{14}$ the authors analyzed a reduced model for the North Atlantic Oscillation (NAO) defined by opposing phases of large atmospheric pressure anomalies over the Atlantic Ocean. This model was obtained from a data-driven clustering method applied to atmospheric reanalysis data. The clustering technique identified the dominant persistent states in the atmosphere and an optimal switching between those states. A reduced model was then constructed using the optimal model parameters and cluster assignments, with the latter corresponding to timedependent switching between states. Each data instance is assigned to one of the clusters however the states themselves are also time dependent and do not correspond to invariant patterns. The model dynamics were then explored through analyzing the directions of local and global growth 
and decay. The authors used an algorithm for computing the covariant Lyapunov vectors (CLVs), which on short time scales produces sets of mixed singular vectors (MSVs). While each regime was found individually to be asymptotically stable, the MSVs showed positive growth rates over short time periods (3 days) associated with structures of a large spatial extent. The characteristics of these MSVs are potentially useful in forecasting in terms of defining the directions in phase space associated with the evolution of large-scale persistent structures of the NAO.

The relative utility of different dynamical vectors in application to ensemble forecast initialization varies in terms of the types of organized structures they project onto during their growth cycle. These structures are often norm dependent and those with smaller spatial scales typically show more rapid development than those with initially larger spatial scales. The respective differences in the spatial scales, growth and convergence rates of SVs, MSVs and CLVs associated with Northern Hemisphere anticyclogenesis over Eurasia are examined in this study through a reduced order linear delay model shown to capture the large-scale switching between various identifiable meta-stable atmospheric states.

Specifically, we focus on identifying the persistent states of atmospheric pressure over the European and western Asian continent. Using the same method as in Quinn, Harries, and O'Kane ${ }^{14}$, we construct a reduced model for persistent atmospheric states over the last four decades. We compute SVs and MSVs associated with each persistent state and compare their relative growth rates and spatial structures when optimized over various time windows. This is then put into the context of extreme event forecasting through a case study of the 2010 Russian heat wave. We discuss the potential utility of such MSVs in application to the initialization of NWP ensembles for medium-range forecasts.

\section{DYNAMICAL SYSTEMS AND PERTURBATION GROWTH}

Consider a dynamical system of the form

$$
\frac{\mathrm{d} \mathbf{x}}{\mathrm{d} t}=\mathbf{F}(\mathbf{x}, t),
$$

where $\mathbf{x}$ is known as the flow that satisfies Eq. 1 for a given initial condition. For spatially discretized flows, Eq. 1 corresponds to a system of ordinary differential equations. Small disturbances $\delta \mathbf{x}$ to the flow $\mathbf{x}$ are governed by the tangent linear dynamics:

$$
\frac{\mathrm{d} \delta \mathbf{x}}{\mathrm{d} t}=\mathbf{A}(\mathbf{x}, t) \delta \mathbf{x}, \quad \text { where } \quad \mathbf{A}(\mathbf{x}, t)=\frac{\partial}{\partial \mathbf{x}} \mathbf{F}(\mathbf{x}, t) .
$$


Here $\mathbf{A}(\mathbf{x}, t)$ is called the tangent linear propagator. The tangent linear propagator can be used to determine the stability properties of the flow, both locally and asymptotically. In particular, one can use compositions of the tangent linear propagator in time to determine the growth rates and directions of growth of perturbations over a specified time window. Over sufficiently long time windows, growth rates define the asymptotic stability of a system and the directions span the subspaces that on average experience the respective growth rate. Rather than asymptotic (or average) behavior, one may in actuality be more interested in local rates and directions of perturbation growth and decay. This is precisely the case in ensemble forecasting. In the following sections we introduce the concepts of local and asymptotic directions of perturbation growth through SVs and CLVs, respectively. We also present another way to measure local growth through the MSVs introduced in Quinn, Harries, and O'Kane ${ }^{14}$. We discuss the computation of each of the vectors and their relations to one another.

\section{A. Singular vectors}

For a matrix M, the SVD is given by

$$
\mathbf{M}=\mathbf{U} \boldsymbol{\Sigma} \mathbf{V}^{*},
$$

where $\mathbf{V}^{*}=\mathbf{V}^{\mathrm{T}}$ when $\mathbf{M}$ is real. The columns of $\mathbf{V}$ are referred to as right-singular vectors $\mathbf{v}_{j}$. These form a non-unique orthonormal basis that gives the directions corresponding to the respective stretching rates $\sigma_{j}$, i.e., the diagonal elements of the matrix $\Sigma$. Singular vectors and their stretching rates are norm-dependent. In other words, the $j^{\text {th }}$ right $\mathrm{SV}\left(\mathbf{v}_{j}\right)$ maximizes the following ratio of $L^{2}$ norms:

$$
\frac{\left\|\mathbf{M v}_{j}\right\|}{\left\|\mathbf{v}_{j}\right\|}=\sigma_{j} .
$$

By definition, the columns of $\mathbf{V}$ are normalised, and therefore $\left\|\mathbf{v}_{j}\right\|=1$. One can choose appropriate weighting for the norms to maximize the stretching if the initial basis is not normalised.

In the context of the tangent linear propagator, one is interested in the SVD of the matrix cocycle over a chosen window $T, \mathcal{A}\left(t_{0}, T\right)$. This can be approximated by a composition matrix of the time-discretized system with time step $\Delta t$ :

$$
\mathcal{A}\left(t_{0}, T\right) \approx \mathbf{A}\left(\mathbf{x}, t_{0}+T\right) \cdot \ldots \cdot \mathbf{A}\left(\mathbf{x}, t_{0}+\Delta t\right) \cdot \mathbf{A}\left(\mathbf{x}, t_{0}\right)
$$

As $\mathbf{x}$ is implicitly dependent on $t$ through Eq. 1, we drop the explicit dependence on $\mathbf{x}$ in the definition of the matrix cocycle $\mathcal{A}$ for simplicity of notation. The right singular vectors obtained 
from the SVD of Eq. 5 correspond to those perturbations with optimal growth rates over the window $t=t_{0}$ to $t=t_{0}+T$ for the chosen norm. Hence, one can identify the perturbation directions associated with the most growth over that time period. This is a method often used when initializing ensemble members in ensemble forecasting (see subsection II D).

\section{B. Covariant Lyapunov vectors}

When one is interested in the asymptotic stability of a system, it is useful to consider Lyapunov exponents and Lyapunov vectors. Lyapunov exponents characterize the long-time growth and decay of a dynamical system. If one or more Lyapunov exponents are positive, then we say that the system is unstable. Otherwise, if all are negative then the system is stable. A Lyapunov exponent $\lambda_{i}$ is defined as follows ${ }^{15}$ :

$$
\lambda_{i}=\lim _{T \rightarrow \infty} \frac{1}{T} \log \left\|\mathcal{A}\left(t_{0}, T\right) \phi\right\| \quad \text { iff } \quad \phi \in \Phi_{i}(\mathbf{x}) \backslash \Phi_{i+1}(\mathbf{x}) .
$$

The subspaces $\Phi_{i}(\mathbf{x})$, known as Oseledets subspaces, decompose the phase space according to the long-time growth and decay rates. In other words, vectors that lie within a given subspace will evolve asymptotically with rate $\lambda_{i}$ forward $\left(-\lambda_{i}\right.$ backwards $)$ in time. These subspaces are covariant with the dynamics and therefore the vectors which span each subspace are known as CLVs. They are norm-independent, give the local directions of the corresponding asymptotic growth and decay in tangent space, and are not required to be orthogonal. While the CLVs are dependent on the underlying flow and therefore provide local information, the directions of growth are optimized for very long time scales.

Since the emergence of numerical algorithms for calculating CLVs ${ }^{16-18}$, the vectors have been used in many studies to determine the hyperbolicity and stability of chaotic systems in applications to convection ${ }^{19,20}$, turbulence ${ }^{21-23}$, and general dissipative systems ${ }^{24-27}$. Additionally the loss of hyperbolicity, or alignment of the CLVs, has been related to critical transitions or regime shifts in systems $^{28-30}$. Recently they have been utilized in the context of ensemble data assimilation through the construction of the background covariance matrix using a subset of the leading CLVs ${ }^{31}$. Most relevant to the study at hand is the application of CLVs to the climate system through the study of stability in quasigeostrophic flow ${ }^{32-34}$, blocking events ${ }^{35}$, and climate teleconnections ${ }^{14}$. 


\section{Mixed singular vectors}

Numerical approximations to the CLVs of a given system are obtained by considering the dynamics of the system over a chosen (sufficiently long) finite time window. As the length of this window is reduced beyond the lower limit for ergodicity, the resulting vectors are no longer CLVs. Instead, these so-called MSVs reflect aspects of the local dynamics, as evidenced by differences in properties such as their alignment and finite-time growth rates ${ }^{14}$. The minimum window length required for the MSVs to converge to CLVs will be different for every system and can roughly be determined through analyzing the finite-time growth rates of the vectors. The averages of the growth rates calculated over many short time intervals should be equal to the asymptotic Lyapunov exponents if the vector is a CLV ${ }^{36}$. We use this property to differentiate between MSVs and CLVs when computing these vectors numerically.

In the following, we compute MSVs and CLVs using Algorithm 2.2 of Froyland et al. ${ }^{18}$ applied over progressively longer time windows. The vector $\phi_{j}^{i}$ will be used to denote the $j^{\text {th }}$ largest MSV or CLV at time $t=t^{i}$. First we choose a push forward $(M)$ and pullback $(N)$ window length, and set of orthogonalization times $\mathbf{N}=\{n, 2 n, \ldots, N\}$ (here the othogonalization time step is $n$ ). The approximation to $\phi_{j}^{i}$ is then obtained utilizing Algorithm 1.

In the subsequent analysis we will use an equal push forward and pullback window $(N=M)$, however they can be chosen independent of one another. The effect of varying the two independently is beyond the scope of this study. The algorithm is initialised using the right singular vectors of the composition matrix from time $t^{i-M}$ to $t^{i}$. Here the optimisation time window is $T=M \Delta t$. The singular vectors are then pushed forward, or evolved, by the tangent linear propagator $M$ time steps. At each orthogonalisation step (including at time $t^{i}$ ), the evolved vectors are enforced to be orthogonal to the leading singular vectors of the forward composition matrix from that time step. This is to avoid collapse onto the leading subspace. In this sense, initial and evolved singular vectors are being "mixed", hence the term MSV to describe the resulting vectors which have not yet converged to CLVs.

\section{Use of singular vectors in ensemble weather forecasting}

The early 1990s saw the introduction of SVs into numerical weather prediction. Mureau, Molteni, and Palmer ${ }^{6}$ used SVs to define the perturbations to the initial states for ensemble 


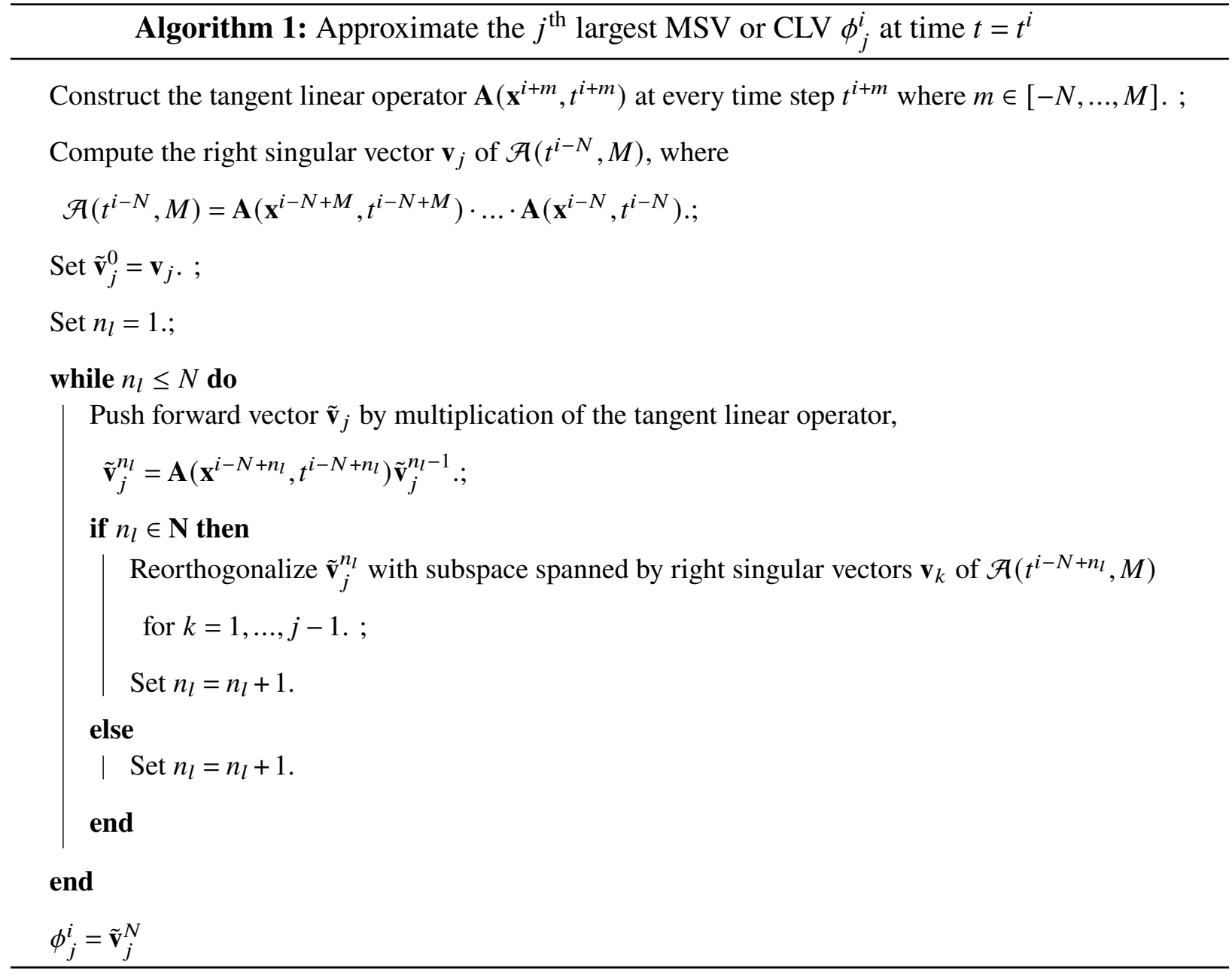

forecasts of the Northern Hemisphere wintertime conditions. The aim was to project onto the fastest growing singular vectors at the time of initialization, such that the ensemble could capture uncertainties due to synoptic scale disturbances in the large-scale background flow. Since then many studies have used SVs to analyze the predictability of weather and climate ${ }^{7,9,37}$ as well as integrated SVs into forecasting systems, most notably at the ECMWF ${ }^{12}$.

We now briefly summarize the implementation of SVs in the ECMWF ensemble prediction system, referring the interested reader to the detailed description in Leutbecher and Palmer ${ }^{13}$. Firstly, optimal perturbations are those which maximize the ratio of the final time to initial time norm. In this sense the norm is weighted by the estimated covariance $\mathbf{C}(t)$ of the perturbations $\mathbf{x} \in \mathbb{R}^{n}$ at time $t:$

$$
\|\mathbf{x}\|_{t}=\mathbf{x}^{\mathrm{T}} \mathbf{C}(t)^{-1} \mathbf{x}
$$

The propagator is constructed for the window of initial to final time which we again notate as $\mathcal{A}\left(t_{0}, T\right)$ for consistency (note in Leutbecher and Palmer ${ }^{13}$ this is defined as $\mathbf{M}$ ). The initial right 
SVs of the propagator maximize

$$
\frac{\left\|\mathcal{A}\left(t_{0}, T\right) \mathbf{v}_{j}\right\|_{t_{0}+T}}{\left\|\mathbf{v}_{j}\right\|_{t_{0}}}=\sigma_{j}, \quad \forall j=1, \ldots, n,
$$

and thus are optimal for the subspace of the estimated initial covariance $\mathbf{C}\left(t_{0}\right)$. The normalized evolved SVs correspond to the left SVs U. The transformed forecast error covariance matrix $\tilde{\mathbf{C}}\left(t_{0}+T\right)=\tilde{\mathbf{U}} \Sigma^{2} \tilde{\mathbf{U}}^{T}$, i.e. the leading initial SVs, evolve into the leading empirical orthogonal function (EOF) decomposition of the normalized forecast error covariance estimate (note that $\boldsymbol{\Sigma}=\mathbf{S}$ in original reference).

For an assumed multivariate Gaussian distribution of the initial errors with zero mean and covariance $\tilde{\mathbf{C}}\left(t_{0}\right)$, the field $\mathbf{x}$ is decomposed into a subspace spanned by only the leading SVs presuming that only the fastest growing errors in the initial distribution are of consequence i.e. directions of greatest rates of expansion in phase space. Unfortunately, the use of initial perturbations constructed in this manner can lead to numerical instabilities. Various strategies are employed to avoid this including discarding the offending perturbations or replacing the underlying Gaussian distribution used in sampling for one with compact support. To enforce zero mean samples of initial perturbations, $+/-$ symmetry can be enforced by generating perturbations in pairs with opposing signs, i.e., including both the perturbation $\delta \mathbf{x}$ and $-\delta \mathbf{x}$ in the sample.

The ECMWF system "mixes" initial and evolved SVs via implementation of a total energy squared norm defined in terms of the zonal and meridional winds, potential temperature, and logarithm of the surface pressure, i.e. the state vector of the atmosphere. These "mixed" SVs are then chosen according to the correspondence of the variance distribution of the SVs to that of the analysis errors (difference between the initial state after data assimilation and verifying observations in model space). For mid-latitude weather forecasts the SVs are optimized for maximum growth over two days, a period for which the tangent linear approximation is assumed to be valid, and a spectral truncation such that synoptic scale disturbances are resolved, roughly equivalent to $\approx 300 \mathrm{~km}$ resolution. The inclusion of evolved SVs in the basis used to generate the mixed SVs allows the initial perturbations to also project onto larger scale structures that exhibit slower growth. SVs specific to particular regions are constructed via multiplying the propagator by a projection operator. In this way varying choices of optimization time, number of leading and evolved SVs initial perturbations, distinct regions in each hemisphere, etc., are made such that specific disturbances and phenomena, for example mid-latitude blocking or tropical cyclones, can be targeted. 
Given the application-specific nature of this empirical approach, it is therefore of practical interest to seek alternative systematic approaches to determining mixed SVs with appropriate properties, i.e optimal structures with physically realistic growth rates, with potential application to NWP.

\section{APPLICATION TO A DATA-DRIVEN REDUCED ATMOSPHERIC MODEL FOR EURASIA}

The data used for this study are the global daily mean $500 \mathrm{hPa}$ geopotential height $\left(Z_{g 500 \mathrm{hPa}}\right)$ fields from the National Centers for Environmental Prediction/National Center for Atmospheric Research (NCEP/NCAR) Reanalysis $1^{38}$. An initial dimension reduction is first performed through an EOF analysis of the daily mean anomalies $Z_{g 500 \mathrm{hPa}}^{\prime}$ with respect to the 1979 to 2018 climatology over the sector $0^{\circ}-90^{\circ} \mathrm{N}, 0^{\circ}-120^{\circ} \mathrm{E}$, with the leading 20 principal components (PCs) retained for the subsequent cluster analysis.

The clustering technique employed here is known as the Finite Element Bounded Variation Vector Autoregressive (FEM-BV-VAR) clustering framework ${ }^{39,40}$. Due to its proven utility in modelling transitional behavior between persistent meta-stable states directly from data, FEM-BVVAR has recently become popular in applications to atmosphere, ocean, and climate data from both

high resolution boundary layer and coarse resolution climate models as well as observations ${ }^{14,41-52}$. Importantly, the method does not rely on any underlying assumptions regarding the statistical stationarity of the data and so is applicable to problems where secular trends are present. In Quinn, Harries, and O'Kane ${ }^{14}$ we describe the FEM-BV-VAR method in detail. Here we briefly describe the method leaving the specifics of the applied fitting procedure to Appendix A. The aim of the method is to fit to a given set of data to a non-stationary stochastic model with time-dependent parameters. This is done by identifying a number of clusters that can each be described by a local stationary vector autoregressive (VAR) model. Using a distance metric specified a priori, the data is assigned an affiliation measure for each cluster at each time step. In order to ensure there is not excessive variation in the affiliations, an upper bound on the total variation norm of the affiliation measure for each cluster state is imposed. This upper bound can be translated to an average persistence of the cluster states. The result of the fit is a set of parameters for the VAR models associated with each cluster, the affiliation sequences $\Gamma_{t}$, and a scalar-valued functional $g$ describing the error of a particular model for a given data instance. 
TABLE I. Persistent events by state

\begin{tabular}{lccc}
\hline \hline & \multicolumn{2}{c}{ Number of events } & Average length (days) \\
\hline State 1 & 60 & 13.27 & 756 \\
State 2 & 12 & 11.33 & 132 \\
State 3 & 3 & 10.50 & 32 \\
\hline
\end{tabular}

To apply the FEM-BV-VAR fitting procedure, one needs to first specify the number of clusters $K$, the order of the VAR models $m$ (also referred to as memory), and the average persistence $p$. As these values are typically not known beforehand, one can run the fitting procedure over many sets of the hyperparameters $(K, m, p)$ and use a chosen criteria to select the optimal model. As noted earlier, we perform the FEM-BV-VAR clustering technique on the reduced space of the leading $20 \mathrm{PCs}$ of the daily mean $500 \mathrm{hPa}$ geopotential height anomalies $\left(Z_{g 500 \mathrm{hPa}}^{\prime}\right)$ for $K \in\{1,2,3\}$, $m \in\{0,1,2,3,4,5\}$, and $p \in\{0,5,10, \ldots, 55,60\}$. The resulting optimal model with the lowest root mean square error using a rolling origin cross-validation has 3 cluster states $(K=3)$, memory of 3 days $(m=3)$ and no persistence condition enforced $(p=0)$.

\section{A. Identifying persistent states}

There is a well established association between persistent anomalies in atmospheric pressure and extreme weather events. For example, particularly intense precipitation events producing strong winds occur over mid-latitude coastal regions of southeastern Australia when isolated low pressure systems (cut-off lows) form in an unstable easterly flow on the northern flank of a slow-moving or blocking anti-cyclone ${ }^{53}$. As we are only interested in studying the local growth rates of the persistent states, we first extract all events such that the model affiliation is constant in one state for a minimum of 10 consecutive days.

In Table I we show the number of these events in the Eurasia sector of the Northern Hemisphere, their average length, and corresponding total number of days each state occurs. State 1 has the most occurrences of persistent events (60) with the longest average duration (approximately 13 days). This is followed by state 2 with 12 events of 11 days on average, while state 3 only experiences 3 events with average length 10.5 days.

To see how the structure of the identified persistent events compares to all events combined, we 
show composites of all days associated with each of the respective persistent events in each given state (Figure 1). The structure of state 1 is dominated by a high pressure anomaly centred over Scandinavia. We also plot for comparison the EOF pattern with the largest pattern correlation, which is EOF 1 at $r=0.94$. This suggests that persistent events in state 1 are associated with the leading mode of variability seen in the data. The composite of persistent events in state 2 shows a pronounced dipolar structure, with the positive and negative anomalies of the dipole having similar magnitude. The negative anomaly also has a large spatial extent, covering the entirety of the European continent. The largest pattern correlation is still with EOF 1, however it is negative and slightly less correlated than state $1(r=-0.71)$. Interestingly, this pattern resembles the negative phase of the NAO when looking at the Atlantic region. While the fitting procedure does not include data from this region, it seems that a negative NAO event drives the persistent widespread negative pressure anomaly over Europe. The composite of persistent events in state 3 appears similar to that of state 1 in that it is characterized by a singular high pressure anomaly except with center over the north west of Europe. This composite has a relatively high pattern correlation with EOF 2. Persistent occurrences of anomalies similar to the state 1 and state 3 composites have been related to heat wave events throughout Europe ${ }^{54,55}$.

\section{B. Comparison of MSVs, SVs, and CLVs}

As persistent events modulate the background state of the atmosphere for an extended period of time, we are interested in the short-term growth behavior during such events. For this we consider the stationary state dynamics for each regime. In Quinn, Harries, and O'Kane ${ }^{14}$ we introduced MSVs to characterize finite-time error growth optimized over short periods. Here we investigate how these vectors compare to SVs calculated for the same composition matrix and the corresponding asymptotic CLVs.

In Appendix B we detail the construction of the reduced dynamical model via the FEM-BV-VAR framework. The resulting tangent linear propagator for the reduced model is given as follows:

$$
\mathbf{A}(\mathbf{x}, t):= \begin{cases}\mathbf{A}_{1} & \text { if } v_{t}=1 \\ \mathbf{A}_{2} & \text { if } v_{t}=2, \\ \mathbf{A}_{3} & \text { if } v_{t}=3 .\end{cases}
$$

Here the propagators $\mathbf{A}_{k}$ for $k \in\{1,2,3\}$ are just constant matrices determined by the parameters 

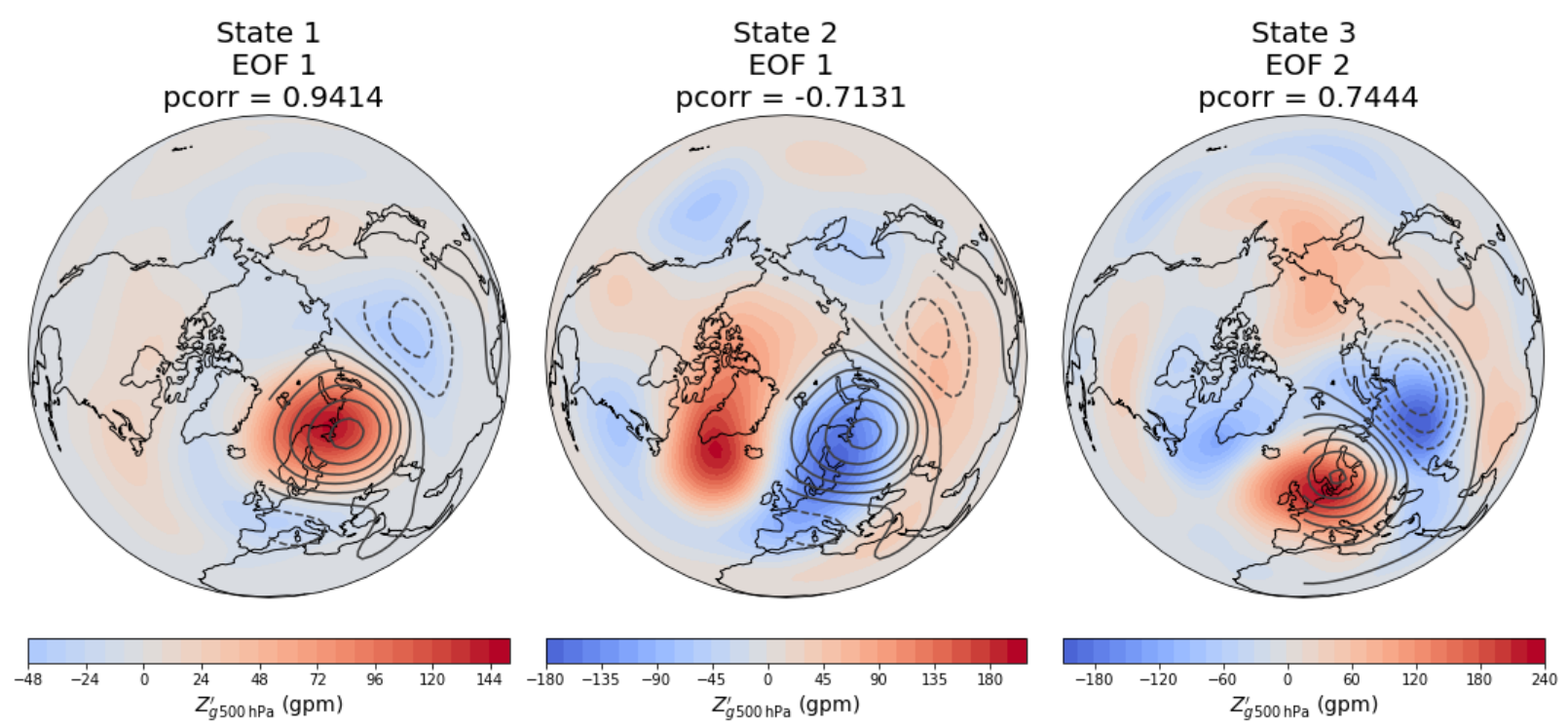

FIG. 1. State affiliation composites for persistent events (10 days or longer) resulting from the FEM-BV-VAR fitting procedure of $500 \mathrm{hPa}$ geopotential height anomalies in the Eurasia region during the period 1979 2018. The parameters for the FEM-BV-VAR fitting procedure are $K=3, m=3$, and $p=0$. The number of samples in each composite is as follows: state 1 - 756 days, state 2 - 132 days, state 3 - 32 days. Contour lines indicate EOF pattern with highest pattern correlation.

corresponding to cluster state $k$, and $v_{t}$ is the Viterbi path denoting the cluster state with the maximum affiliation value at time $t$ (see Appendix A for more details). The composition matrix (Eq. 5) over a given window $T$ is then just given by multiplication of the corresponding sequence of constant matrices.

To investigate the dynamics of persistent states we can simply analyze the stationary state dynamics. For this we use compositions of a given cluster's constant propagators from Eq. 9 for various window lengths. As we are interested in the finite-time growth over short periods, we compute sets of MSVs for each of the stationary states. Additionally we compute sets of SVs over the same time windows. More specifically, if a push forward of $M$ is used for Algorithm 1, then the SVs will be calculated for the composition matrix $\mathcal{A}\left(t_{0}-M, M\right)$ (note here that since we are working with constant dynamics the $t_{0}$ is irrelevant). We also compute the asymptotic Lyapunov exponents of each stationary state using the $\mathrm{QR}$ algorithm ${ }^{56}$.

Figure 2 shows the one-day growth rates for the leading 5 MSVs and SVs, as well as the 5 leading 

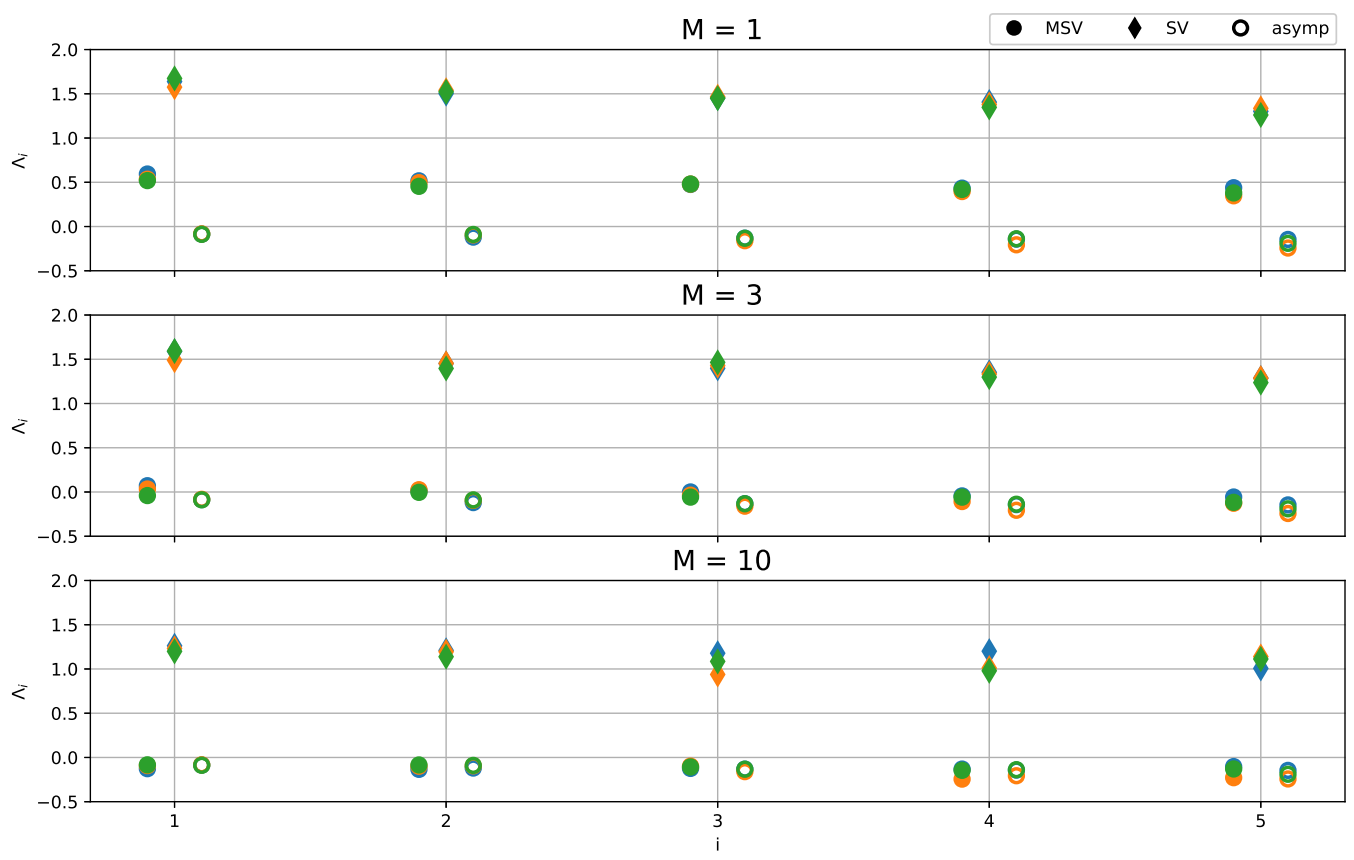

FIG. 2. Growth rates over one time step of MSVs and SVs for stationary states 1 (blue), 2 (orange), and 3 (green). The MSVs and SVs are calculated over a window of 1, 3, and 10 days. The asymptotic growth rates for each stationary state are shown for comparison.

asymptotic Lyapunov exponents. The growth rates of the MSVs approach the asymptotic growth rates as the push forward is increased. This is expected as by definition the MSVs should converge to CLVs as $M$ increases in Algorithm 1. This means the MSVs will approach the asymptotic subspaces on which the Lyapunov exponents are defined. On the other hand, the leading SVs retain high growth rates even as the push forward is increased. It is known that SVs can commonly grow much faster than Lyapunov exponent growth ${ }^{7,12}$.

In addition to the growth rates, we are also interested in the atmospheric patterns onto which the vectors project. Figure 3 shows the leading SV, MSV, and their difference for stationary state 1. Both vectors, SV and MSV, result in large-scale patterns for the short push forwards with only small offsets in the anomaly centers. As the push forward increases, however, the patterns begin to diverge. The SV develops small-scale anomalies while the MSV converges further to a large-scale pattern similar to those resulting from the short push forwards. The leading modes are meant to represent the patterns with most growth (or least decay) over the push forward window. As 

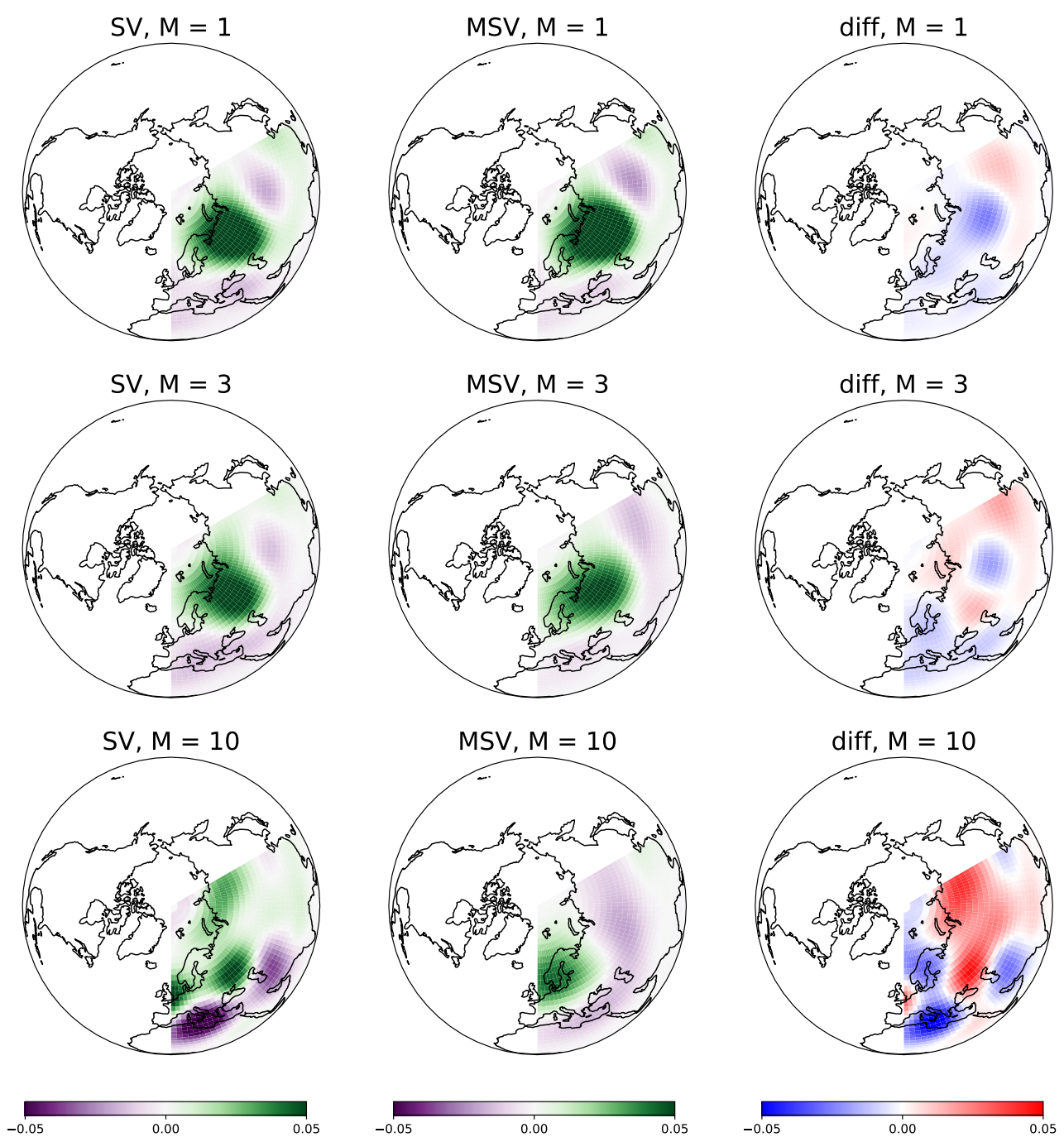

FIG. 3. Projections of leading MSVs and SVs for stationary state 1 calculated using different push forward steps. The MSVs and SVs are calculated over a window of 1, 3, and 10 days. The difference (SV-MSV) is also shown for comparison.

the dynamics at hand are stationary and stable, we know that the model will not have growing modes for long push forwards and that the least-decaying mode will be similar to the composite of persistent events in a given state shown in Figure 1. The emergence of unstable small-scale structures for long push forwards gives further evidence that the leading SVs are not projecting onto the physically relevant atmospheric patterns for the time periods of interest. 


\section{CASE STUDY: 2010 RUSSIAN HEAT WAVE}

To provide a practical example of the utility of MSVs as potential initial perturbations for ensemble forecasting, we now turn our attention to a specific extreme event, namely the Russian heat wave (RHW) during summer 2010. This heat wave is considered to have taken place over the two month period from mid-June to mid-August, with its peak impacts in late July and early August $^{57-59}$. The event is attributed to a strong and persistent atmospheric blocking event centred over Moscow ${ }^{60}$. As blocking is associated with persistent large-scale high pressure anomalies, we would expect the Russian heat wave to be represented in our model by persistence and recurrence to a pattern closely associated with the previously identified metastable state 1 .

In order to investigate the RHW we first identify the relevant dates of impact using two standard heat wave indices. The first is a generally accepted definition from the World Meteorological Organization (WMO), which requires 5 or more consecutive days in which the maximum temperature exceeds the climatological average maximum temperature by $5^{\circ} \mathrm{C}$. The second index, introduced by Russo et al. ${ }^{61}$, is known as the Heat Wave Magnitude Index (HWMI) and is defined by 3 or more consecutive days in which the maximum temperature is in the $90^{\text {th }}$ percentile of climatological daily maxima within a 31 day window of the year. We calculate these two indices for a box centered over Moscow $\left(52.5^{\circ} \mathrm{N}-57.5^{\circ} \mathrm{N}, 35^{\circ} \mathrm{E}-40^{\circ} \mathrm{E}\right)$. Figure 4 shows the resulting WMO and HWMI heat wave indicators along with the affiliation sequence for our reduced model. We see that both indicate a heat wave beginning on 21 June 2010, coincident with the onset of a semi-persistent state 1 (5 days). There is then a brief excursion (3 days) to the other two states before returning to another persistent state 1 event. Although the HWMI shows a brief interruption to the heat wave around the beginning of July, both indices identify the same time period for the heat wave. Throughout the duration of the heat wave we observe that the reduced model has a preference for state 1 , which is characterized by a persistent positive height anomaly over Moscow, with infrequent brief excursions to one of the other two states of never more than 3 consecutive days. This aligns with the conjecture that persistent state 1 events correspond to heat wave events in Europe.

We have previously described the use of combinations of initial and evolved SVs at the $\mathrm{ECMWF}^{12,13}$. Here we are interested in whether or not the systematically generated MSVs can also adequately characterize the dynamics and error growth, and hence predictability, when optimized over windows appropriate to medium-range weather forecasting (days to weeks). We take a start date of 15 July 2010 such that the heat wave has been well established and continues 


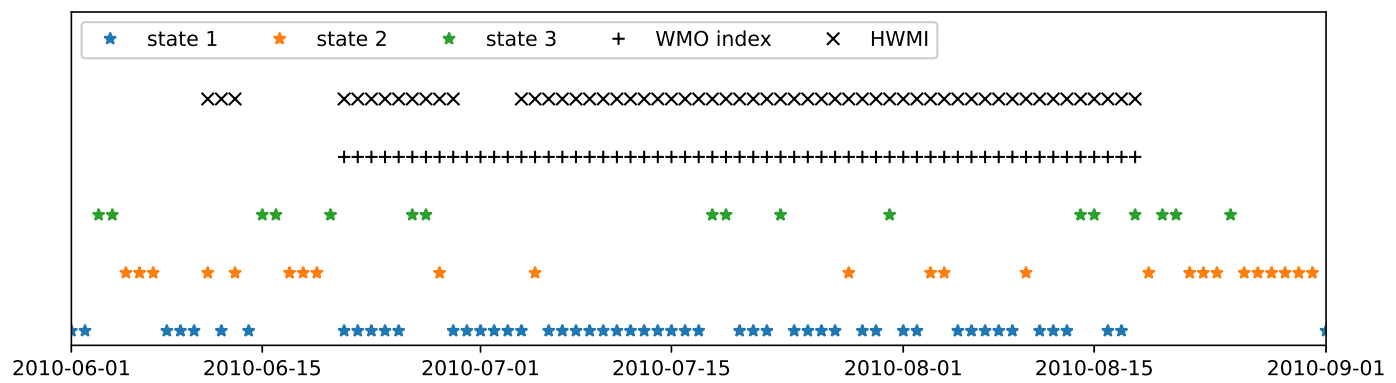

FIG. 4. Heat wave indices and state affiliation sequence for the reduced model Eq. (B1) in JJA 2010. The HWMI is indicated by $\times$ 's and the WMO index by +'s. FEM-BV-VAR metastable states are colored as: state $1 \star$, state $2 \star$ and state $3 \star$ respectively.

for a period of more than 10 days (according to Figure 4), and we compare the behavior of both SVs and MSVs on forward propagation.

We again consider the leading growth rates first, which are plotted in Figure 5. As with the stationary case, the SVs start out with much larger growth rates than the MSVs. The growth rates remain positive over 4 days for most of the push forward values. Even for a very long window of 100 days, which theoretically should be optimizing the structures which grow over that time period, the growth is faster than that of the MSVs optimized over 1 day. Additionally, given that our model is globally stable, we would not expect such growth for structures optimized over such a long window. We know that the growth of any arbitrary vector will converge to the leading Lyapunov exponent after a sufficient amount of time. We therefore show the growth of the leading SVs and MSVs out to 16 days to illustrate the relative behavior of this convergence. We see that the shorter push forward windows show a slower convergence to that growth and still underestimate it by day 16 . For a very long window of 100 days, we see that the MSV is already on the asymptotic subspace (as expected), while the SV takes a few days to approach the subspace and still has not quite converged by the end of the 16 days. As can be seen by the values of the asymptotic Lyapunov exponents in Figure 5, our model is globally stable. We therefore expect that as perturbations are optimized over longer windows, those perturbation vectors will approach an increasingly stable subspace. This indicates that the MSVs are better able to characterize error growth optimized over particular finite time windows, however we observe that the SVs consistently initialize in a relatively unstable subspace.

In order to give more insight on the spatial patterns of the vectors, their propagation consistent 
GR 1
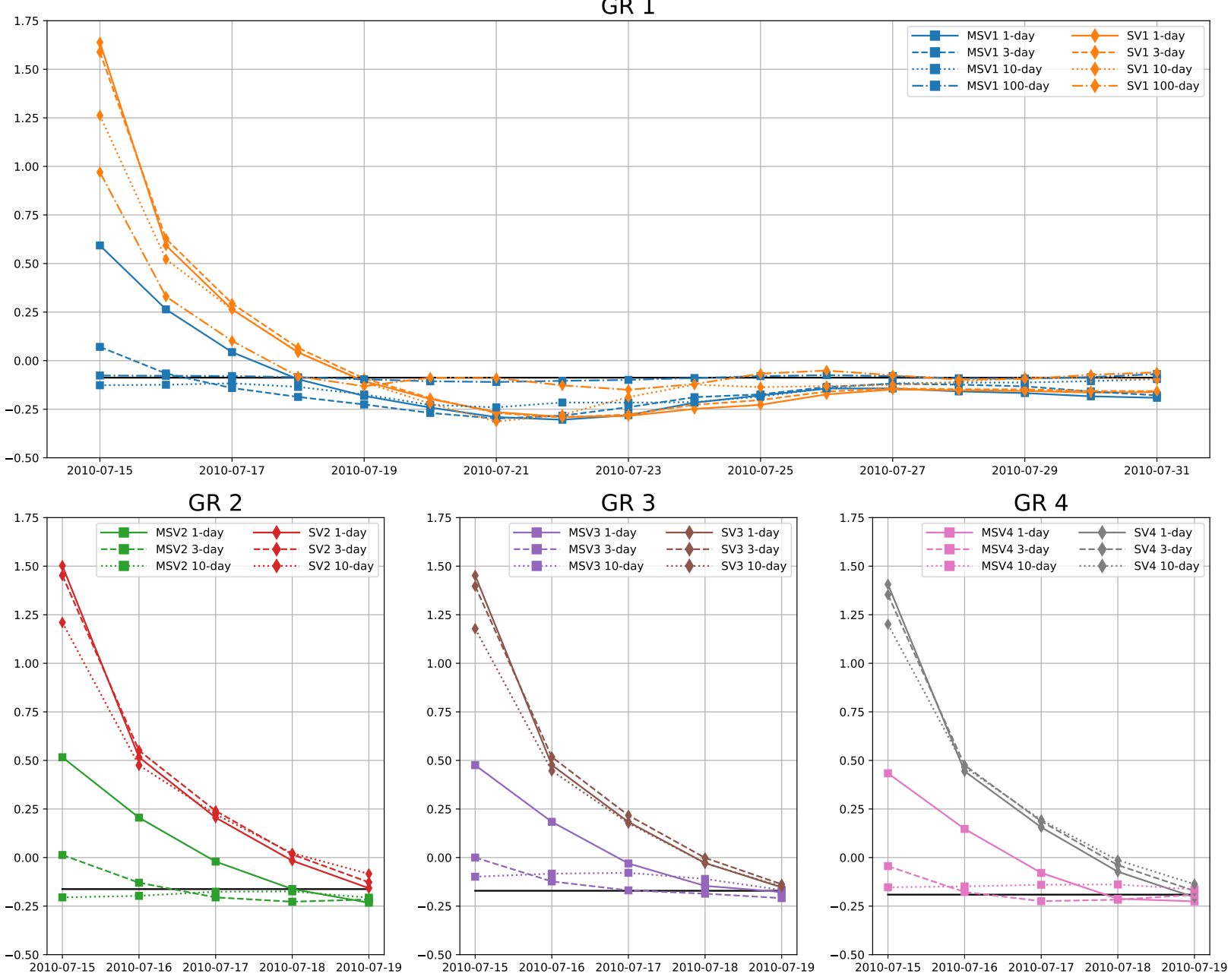

FIG. 5. Growth rates of the leading four SVs (diamonds) and MSVs (squares) under forward propagation during the 2010 Russian heat wave. Solid black lines show the corresponding asymptotic Lyapunov exponents for the full time series. The vectors are propagated using the tangent linear propagator Eq. (B5) as constructed using the affiliation sequence starting from 15 July 2010.

with the growth rates described earlier in Figure 5, and relationship to the geographical location of the observed RHW structure, in Figure 6, we plot the four leading SVs and MSVs on 15 July 2010 calculated for an optimization period of 10 days. Specifically, we show the patterns of the initial SV's 1,2,3 \& 4 and MSV's 1,2,3 \& 4 and their subsequent patterns after they have been pushed forward (evolved) 5 days by the tangent linear propagator. These evolved patterns correspond to the growth rates on 19 July 2010 described in Figure 5.

We first observe that in the initial vectors there are qualitatively similar differences between each SV and MSV. The SVs contain smaller-scale features that do not represent typical structures 

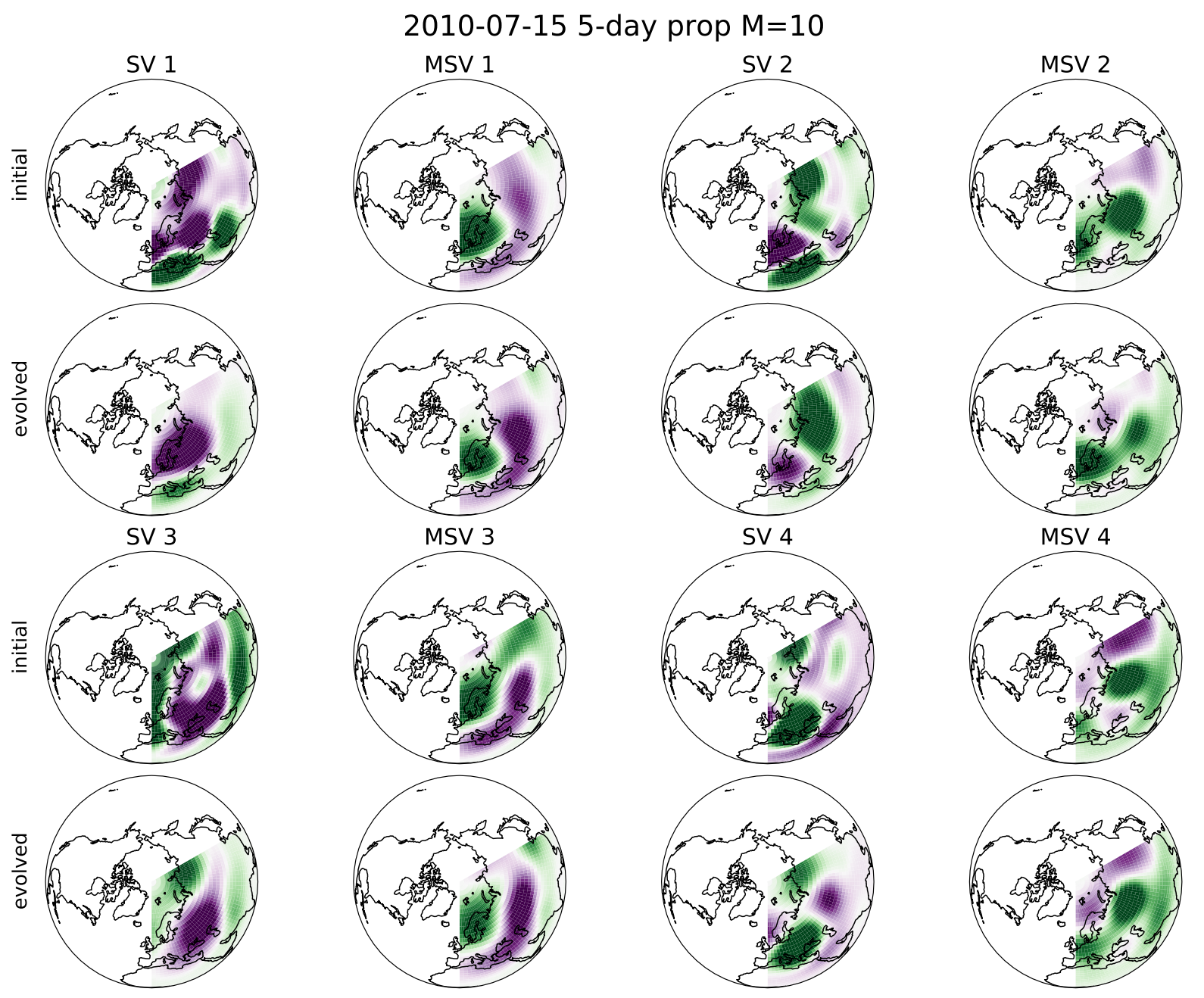

FIG. 6. Projections of initial and evolved leading 10-day SVs and MSVs during the 2010 Russian heat wave. The initial vectors are computed on the 15 July 2010 and propagated forward 5 days using the tangent linear propagator Eq. (B5) and the affiliation sequence Eq. (B1).

of 10-day atmospheric anomalies. The MSVs, on the other hand, contain structures that resemble 10-day averaged pressure anomalies. These have larger spatial extent and either meridional or zonal orientation of pressure differences, which can be linked to barotropic and baroclinic instabilities in the background flow. In particular, we see that MSVs 2 and 4 identify high pressure anomalies sitting in roughly the right location to bring warm air northward towards the Moscow region, while MSVs 1 and 3 isolate the zonal flow through the low pressure band across southeastern Europe and central Asia.

On propagating the vectors we see that the SVs eventually converge to patterns with larger spatial structures. However, the location of the large-scale structures differs from that of the 
evolved MSVs, and the connection between the initial and evolved SV patterns is unclear. The MSVs appear to evolve as a propagation of their initial states with some growth and decay of the large-scale features. We observe that MSV 1 appears to develop a dipolar structure with a high pressure anomaly over Scandanavia similar to the composite pattern of state 3 (we note that Figure 4 indicates the model is in state 3 on 18 and 19 July). The structures associated with the heat wave in MSVs 2 and 4 remain, albeit smaller for MSV 2, as the two vectors begin to align with a similar spatial pattern. This could potentially be used as evidence for the continuation of the heat wave despite the leading vector developing an opposing anomaly in that region.

In relation to medium-range weather forecasting, the MSVs provide initial conditions isolating large-scale features and their subsequent evolution, and therefore may be optimal to use over SVs in this context.

\section{SUMMARY AND DISCUSSION}

The study by Quinn, Harries, and O' Kane ${ }^{14}$ showed the utility of the FEM-BV-VAR methodology ${ }^{39}$ to generate a reduced order linear stochastic model that is computationally tractable and yet of sufficient complexity that it can effectively capture the underlying dynamics of transitions between persistent states indicative of the North Atlantic Oscillation (NAO) and the Atlantic Ridge (AR) in the mid-latitude Northern Hemisphere troposphere. They further showed that the characteristic dynamics of the associated matrix cocycle ${ }^{18}$ can give a deeper insight to the role of local instabilities in the onset and decay, as well as persistence and frequency, of those aforementioned important weather patterns. Here we are interested in the utility of the approach in the context of atmospheric blocking over Eurasia, a region where anomalously long-lived and persistent positive geopotential height structures can manifest extreme heat wave events. On the basis of our results, it is readily apparent that the non-parametric data-driven reduced order linear model obtained is capable of not simply representing the climatological characteristics of blocking over Eurasia, but also the dynamics of particular events and specifically the 2010 RHW.

We have discussed the use of SVs as deployed in the ECMWF weather forecast system, noting the combination of initial and evolved SVs through the implementation of a total energy norm. We note the complexity of that approach required to obtain the high degree of skill for global weather systems for which the ECMWF system is renowned. Our results show that the MSVs generated from a matrix cocycle determined for a suitably chosen push forward time allows a consistent 
mathematical framework for generating initial structures that are capable of characterizing the information relevant to the cycles of persistent coherent structures in the troposphere. In the context of the 2010 RHW, we have shown the relationship between initial and evolved SVs and MSVs optimized over a 10-day period, where the latter are more indicative of a given persistent event and are a natural choice to generate dynamically relevant initial structures with growth rates characteristic of local disturbances on the timescales of large-scale synoptic weather systems.

Another challenge that emerges in ensemble NWP is the number of ensemble members needed to adequately sample the uncertainty in model evolution. This uncertainty can be related to the unstable subspace of the model, or rather the directions in which the model phase space is expanding. In the context of data assimilation in the unstable subspace determined by a simple (1-dimensional in space) multiscale paradigm model, Quinn, O'Kane, and Kitsios ${ }^{31}$ showed that the attractor dimension, as determined by the Kaplan-Yorke dimension based on the system's Lyapunov exponents, can be effectively employed to determine both the global and local stability properties of a dynamical system. They further showed that the time-dependent local attractor dimension, also associated with the fluctuating alignment of the corresponding dynamical vectors in time, gives the required rank for the background (forecast) covariances at any given analysis time for ensemble data assimilation and forecast initialization. Here we show that the insights gained from the simple paradigm model hold when applied to high dimensional reconstructed (reanalysis) weather data. We observe that varying the optimization window for the MSVs results in a variable number of unstable (and neutral) local directions for each time window of interest. The close association between local dimension and the number of dynamical vectors required to capture the growth rates of the relevant local spatio-temporal instability means that the local dimension not only defines the unstable subspace itself but is also a time-dependent lower bound on the number of directions required to span the local manifold.

While much of climate science is directed towards developing general circulation models which represent the dynamics of the ocean, atmosphere, land, sea ice and cryosphere at ever increasing resolutions and complexity, there is a recognition in the geophysical mathematics community of the increased importance of furthering an understanding of the fundamental dynamical and statistical properties of the climate system. This is often done via reduced order models that capture the essential dynamics of the climate modes and with the ultimate aim of characterizing the causal relationships between these teleconnections, their regime dependencies, and their response to anthropogenic changes in the forcing. Nevertheless, many of the challenges that faced the early 
pioneers of numerical weather prediction remain in the climate models of increasing complexity. Chief among these is the accurate and efficient characterization of local disturbances and their spatio-temporal growth. The fact that such disturbances are generated by nonlinear dynamics on a local unstable subspace in the absence of hyperbolicity is a severe challenge requiring advances across a range of mathematical disciplines. Beyond weather to seasonal prediction and multi-year climate forecasts where various domains of the planetary system whose disparate temporal scales are coupled, operational data assimilation and (ensemble) prediction systems with the ability to project onto the slow scales of relevant coupled instabilities while simultaneously incorporating the required stochastic representation of fast forcing scales remains an unsolved problem. That said, there is an emerging body of theoretical work to which the work at hand contributes, focused on developing a deeper understanding of variability and predictability in the climate system.

\section{ACKNOWLEDGMENTS}

CQ and TJO were supported by the Australian Commonwealth Scientific and Industrial Research Organisation (CSIRO) Decadal Climate Forecasting Project (https://research.csiro.au/ $\mathrm{dfp}$ ). DH was supported by the CSIRO through a ResearchPlus postdoctoral fellowship. The EOF analysis was implemented using the scikit-learn Python package ${ }^{62}$, and plots were generated using the Python package Matplotlib ${ }^{63}$.

\section{DATA AVAILABILITY STATEMENT}

The NCEP-NCAR reanalysis output used is provided by the NOAA/OAR/ESRL PSL, Boulder, Colorado, and may be accessed at https://psl.noaa.gov/data/reanalysis/reanalysis.shtml. All source code used to perform the analyses presented in this study may be found at https://doi.org/10.5281/zenodo.4035644.

\section{Appendix A: FEM-BV-VAR specifics}

The Finite Element Bounded Variation Vector Auto-Regressive method developed by Horenko 39 as also described in Metzner, Putzig, and Horenko ${ }^{40}$, is a data-driven clustering method which extracts metastable states and the associated switching sequence between the states. Each of the 
states is assumed to be defined by a locally stationary linear VAR model:

$$
\mathbf{x}_{t}=\mu^{k}+\sum_{i=1}^{m} \mathbf{P}_{i}^{k} \mathbf{x}_{t-i}+\epsilon_{t} \quad \text { for } k=1, \ldots, K .
$$

Here we have introduced two hyperparameters, $K$ and $m$. The first refers to the number of metastable regimes while the second defines the memory dependence of the VAR models. The model parameters $\mu^{k}$ and $\mathbf{P}^{k}$ are constant within each cluster state as is the covariance matrix $\Sigma(t)$. $\epsilon_{t}$ is random noise required to initiate transitions between states.

We seek to apply a regularized variational minimization of a scalar-valued functional describing the error $g\left(x_{t}, \Theta^{k}\right)$ of a particular model for a given observational data instance $x(t)$ characterized by a time dependent set of model parameters $\Theta(t)$. We first construct a distance functional for a given time $t$ :

$$
\mathbf{L}(\Theta, \Gamma)=\sum_{k=1}^{K} \gamma_{k}(t) g\left(x_{t}, \Theta^{k}\right),
$$

where $\Theta^{k}=\left[\mu^{k}, \mathbf{P}_{1}^{k}, \ldots, \mathbf{P}_{m}^{k}\right]$ and $\gamma_{k}(t)$ is called the affiliation to a given cluster $k$. The affiliations $\Gamma(t)=\left(\gamma_{1}(t), \ldots, \gamma_{K}(t)\right)$ are subject to the constraints

$$
\begin{aligned}
& \sum_{k=1}^{K} \gamma_{i}(t)=1, \quad \forall t \in[0, T], \\
& \gamma_{k}(t) \geq 0, \quad \forall t \in[0, T], \forall k=1, \ldots, K,
\end{aligned}
$$

where $\Theta(t)=\sum_{k=1}^{K} \gamma_{k}(t) \Theta^{k}$ and

$$
\begin{gathered}
\left|\gamma_{k}(\cdot)\right|_{B V}=\sum_{t=2}^{T}\left|\gamma_{k}(t+\tau)-\gamma_{k}(t)\right| \leq N_{C}, \\
\forall k=1, \ldots, K
\end{gathered}
$$

The regularization parameter $N_{C}$ controls the number of transitions between different clusters in time and constrains the regime-switching process to be of bounded variation (BV) in time, thereby making the temporal change of the inferred model parameters $\Theta(t)$ more or less persistent. This results in an average persistence hyperparameter $p$ calculated through $p=\frac{T}{N_{C}+1}$.

In the FEM-BV methodology, finite element methods are employed in the numerical representation of indicator functions $\gamma_{k}(t)$ for the time domain of applicability of different models from a common model class. Model class is defined by the choice of the particular analytical form of the error function $g\left(x_{t}, \Theta_{k}\right)$. The choice of the model class depends on the type of data and corresponding 
system being analyzed. The explicit VAR model considered here is $x_{t}=\mu(t)+\sum_{i=1}^{m} \mathbf{P}_{i}(t) x_{t-i \tau}+\epsilon_{t}$, where the model error functional is

$$
g\left(x_{t}, \Theta_{k}\right)=\left\|x_{t}-\left(\mu(t)+\sum_{i=1}^{m} \mathbf{P}_{i}^{k}(t) x_{t-i \tau}\right)\right\|_{2}^{2} .
$$

Here we optimize for fixed $\Theta$ followed by optimizing $\Theta$ for a fixed $\Gamma$. This procedure is repeated iteratively, until the change in $L(\Theta, \Gamma)$ is less than some small pre-determined threshold, resulting in monotonic minimization of $L$. In summary, we have used an adaptive finite element method for the numerical minimization of the FEM-BV Eq. A2. The number of different meta-stable states, or clusters $K$, and the model parameters chosen within these regimes, such as memory depth $m$, the indicator functions $\gamma_{k}(\cdot)$ signaling activation of the respective models, are all determined simultaneously in a global optimization procedure. The optimization yields a judicious compromise between low residuals, reproducing the data of a training set on the one hand, and the demand for the smallest-possible overall number of free parameters of the complete model on the other.

\section{Appendix B: Model specifics}

Given the results of the FEM-BV-VAR fitting procedure outlined in Appendix A, we can construct a reduced time-dependent model for the dynamics. We first assign each time step to a cluster state determined by the maximum affiliation at that time:

$$
v_{t}=\underset{k}{\arg \max }[\Gamma(t)] \quad \text { for } \quad k \in\{1,2,3\} .
$$

Eq. B1 is known as the Viterbi path in Hidden Markov Model literature. We can then use the Viterbi path and the model parameters to define the VAR model at each time step through

$$
\mathbf{x}_{t}=\mu^{\left(v_{t}\right)}+\sum_{i=1}^{m} \mathbf{P}_{i}^{\left(v_{t}\right)} \mathbf{x}_{t-i}
$$

Note that we have dropped the stochastic element of the VAR model as we are interested in evaluating the deterministic dynamics.

By extending the state space to account for the memory in the model, we construct a dimension $60(3 \times 20$ PCs $)$ discrete linear map whose tangent dynamics are governed by

$$
\left[\begin{array}{c}
\mathbf{x}_{t} \\
\mathbf{x}_{t-1} \\
\mathbf{x}_{t-2}
\end{array}\right]=\left[\begin{array}{ccc}
\mathbf{P}_{1}^{\left(v_{t}\right)} & \mathbf{P}_{2}^{\left(v_{t}\right)} & \mathbf{P}_{3}^{\left(v_{t}\right)} \\
\mathbf{I} & \mathbf{0} & \mathbf{0} \\
\mathbf{0} & \mathbf{I} & \mathbf{0}
\end{array}\right]\left[\begin{array}{l}
\mathbf{x}_{t-1} \\
\mathbf{x}_{t-1} \\
\mathbf{x}_{t-3}
\end{array}\right] .
$$


Our tangent linear propagator is then given as

$$
\mathbf{A}(\mathbf{x}, t):=\left[\begin{array}{ccc}
\mathbf{P}_{1}^{\left(v_{t}\right)} & \mathbf{P}_{2}^{\left(v_{t}\right)} & \mathbf{P}_{3}^{\left(v_{t}\right)} \\
\mathbf{I} & \mathbf{0} & \mathbf{0} \\
\mathbf{0} & \mathbf{I} & \mathbf{0}
\end{array}\right]
$$

or alternatively,

$$
\mathbf{A}_{k}=\left[\begin{array}{ccc}
\mathbf{P}_{1}^{k} & \mathbf{P}_{2}^{k} & \mathbf{P}_{3}^{k} \\
\mathbf{I} & \mathbf{0} & \mathbf{0} \\
\mathbf{0} & \mathbf{I} & \mathbf{0}
\end{array}\right] \quad \text { for } \quad v_{t}=k
$$

\section{REFERENCES}

${ }^{1}$ E. N. Lorenz, "Deterministic nonperiodic flow," Journal of atmospheric sciences 20, 130-141 (1963).

${ }^{2}$ T. J. O'Kane and J. S. Frederiksen, “A comparison of statistical dynamical and ensemble prediction methods during blocking," J. Atmos. Sci. 65, 426-446 (2008).

${ }^{3}$ E. N. Lorenz, “A study of the predictability of a 28 -variable atmospheric model," Tellus 17, 321-333 (1965).

${ }^{4}$ E. N. Lorenz, “Atmospheric predictability as revealed by naturally occurring analogues," Journal of Atmospheric Sciences 26, 636-646 (1969).

${ }^{5}$ N. A. Phillips, "Energy transformations and meridional circulations associated with simple baroclinic waves in a two-level, quasi-geostrophic model,” Tellus VI, 273-286 (1954).

${ }^{6}$ R. Mureau, F. Molteni, and T. Palmer, "Ensemble prediction using dynamically conditioned perturbations," Quarterly Journal of the Royal Meteorological Society 119, 299-323 (1993).

${ }^{7}$ F. Molteni and T. Palmer, "Predictability and finite-time instability of the northern winter circulation," Quarterly Journal of the Royal Meteorological Society 119, 269-298 (1993).

${ }^{8}$ R. Buizza, J. Tribbia, F. Molteni, and T. Palmer, "Computation of optimal unstable structures for a numerical weather prediction model," Tellus A 45, 388-407 (1993).

${ }^{9}$ T. N. Palmer, R. Buizza, F. Molteni, Y.-Q. Chen, and S. Corti, "Singular vectors and the predictability of weather and climate," Philosophical Transactions of the Royal Society of London. Series A: Physical and Engineering Sciences 348, 459-475 (1994).

${ }^{10}$ D. Hartmann, R. Buizza, and T. N. Palmer, "Singular vectors: The effect of spatial scale on linear growth of disturbances," Journal of Atmospheric Sciences 52, 3885-3894 (1995). 
${ }^{11}$ R. Buizza and T. N. Palmer, "The singular-vector structure of the atmospheric global circulation," Journal of the Atmospheric Sciences 52, 1434-1456 (1995).

${ }^{12}$ F. Molteni, R. Buizza, T. N. Palmer, and T. Petroliagis, “The ecmwf ensemble prediction system: Methodology and validation," Quarterly journal of the royal meteorological society 122, 73-119 (1996).

${ }^{13}$ M. Leutbecher and T. N. Palmer, "Ensemble forecasting," Journal of computational physics 227, 3515-3539 (2008).

${ }^{14}$ C. Quinn, D. Harries, and T. J. O'Kane, “Dynamical analysis of a reduced model for the north atlantic oscillation,” Journal of the Atmospheric Sciences ?, ?-? (2021).

${ }^{15}$ V. I. Oseledets, “A multiplicative ergodic theorem. characteristic ljapunov, exponents of dynamical systems," Trudy Moskovskogo Matematicheskogo Obshchestva 19, 179-210 (1968).

${ }^{16}$ C. L. Wolfe and R. M. Samelson, “An efficient method for recovering lyapunov vectors from singular vectors," Tellus A: Dynamic Meteorology and Oceanography 59, 355-366 (2007).

${ }^{17}$ F. Ginelli, P. Poggi, A. Turchi, H. Chaté, R. Livi, and A. Politi, "Characterizing dynamics with covariant lyapunov vectors," Physical review letters 99, 130601 (2007).

${ }^{18}$ G. Froyland, T. Hüls, G. P. Morriss, and T. M. Watson, "Computing covariant lyapunov vectors, oseledets vectors, and dichotomy projectors: A comparative numerical study," Physica D: Nonlinear Phenomena 247, 18-39 (2013).

${ }^{19}$ M. Xu and M. R. Paul, "Covariant lyapunov vectors of chaotic rayleigh-bénard convection," Physical Review E 93, 062208 (2016).

${ }^{20}$ M. Xu and M. Paul, "Spatiotemporal dynamics of the covariant lyapunov vectors of chaotic convection," Physical Review E 97, 032216 (2018).

${ }^{21}$ M. Inubushi, M. U. Kobayashi, S.-i. Takehiro, and M. Yamada, "Covariant lyapunov analysis of chaotic kolmogorov flows," Physical Review E 85, 016331 (2012).

${ }^{22}$ M. Inubushi, M. U. Kobayashi, S.-i. Takehiro, and M. Yamada, "Covariant lyapunov analysis of chaotic kolmogorov flows and time-correlation function," Procedia IUTAM 5, 244-248 (2012).

${ }^{23}$ M. Inubushi, S.-i. Takehiro, and M. Yamada, "Regeneration cycle and the covariant lyapunov vectors in a minimal wall turbulence," Physical review E 92, 023022 (2015).

${ }^{24}$ P. V. Kuptsov and S. P. Kuznetsov, "Violation of hyperbolicity in a diffusive medium with local hyperbolic attractor,” Physical Review E 80, 016205 (2009).

${ }^{25}$ H.-l. Yang, K. A. Takeuchi, F. Ginelli, H. Chaté, and G. Radons, "Hyperbolicity and the effective dimension of spatially extended dissipative systems," Physical review letters 102, 074102 (2009). 
${ }^{26}$ K. A. Takeuchi, H.-1. Yang, F. Ginelli, G. Radons, and H. Chaté, "Hyperbolic decoupling of tangent space and effective dimension of dissipative systems," Physical Review E 84, 046214 (2011).

${ }^{27}$ H.-l. Yang and G. Radons, “Geometry of inertial manifolds probed via a lyapunov projection method," Physical review letters 108, 154101 (2012).

${ }^{28} \mathrm{M}$. W. Beims and J. A. Gallas, “Alignment of lyapunov vectors: A quantitative criterion to predict catastrophes?" Scientific reports 6, 1-7 (2016).

${ }^{29}$ N. Sharafi, M. Timme, and S. Hallerberg, "Critical transitions and perturbation growth directions," Physical Review E 96, 032220 (2017).

${ }^{30}$ E. L. Brugnago, J. A. Gallas, and M. W. Beims, "Machine learning, alignment of covariant lyapunov vectors, and predictability in rikitake's geomagnetic dynamo model," Chaos: An Interdisciplinary Journal of Nonlinear Science 30, 083106 (2020).

${ }^{31}$ C. Quinn, T. J. O'Kane, and V. Kitsios, “Application of a local attractor dimension to reduced space strongly coupled data assimilation for chaotic multiscale systems," Nonlinear Processes in Geophysics 27, 51-74 (2020).

${ }^{32}$ S. Schubert and V. Lucarini, "Covariant lyapunov vectors of a quasi-geostrophic baroclinic model: analysis of instabilities and feedbacks," Quarterly Journal of the Royal Meteorological Society 141, 3040-3055 (2015).

${ }^{33}$ A. Gritsun and V. Lucarini, "Fluctuations, response, and resonances in a simple atmospheric model,” Physica D: Nonlinear Phenomena 349, 62-76 (2017).

${ }^{34} \mathrm{~S}$. Vannitsem and V. Lucarini, "Statistical and dynamical properties of covariant lyapunov vectors in a coupled atmosphere-ocean model-multiscale effects, geometric degeneracy, and error dynamics," Journal of Physics A: Mathematical and Theoretical 49, 224001 (2016).

${ }^{35}$ S. Schubert and V. Lucarini, "Dynamical analysis of blocking events: spatial and temporal fluctuations of covariant lyapunov vectors," Quarterly Journal of the Royal Meteorological Society 142, 2143-2158 (2016).

${ }^{36} \mathrm{P}$. V. Kuptsov and U. Parlitz, "Theory and computation of covariant lyapunov vectors,” Journal of nonlinear science 22, 727-762 (2012).

${ }^{37}$ R. Buizza, R. Gelaro, F. Molteni, and T. Palmer, "The impact of increased resolution on predictability studies with singular vectors," Quarterly Journal of the Royal Meteorological Society 123, 1007-1033 (1997).

${ }^{38}$ E. Kalnay, M. Kanamitsu, R. Kistler, W. Collins, D. Deaven, L. Gandin, M. Iredell, S. Saha, 
G. White, J. Woollen, et al., “The ncep/ncar 40-year reanalysis project,” Bulletin of the American meteorological Society 77, 437-472 (1996).

${ }^{39}$ I. Horenko, "On the identification of nonstationary factor models and their application to atmospheric data analysis,” Journal of the Atmospheric Sciences 67, 1559-1574 (2010).

${ }^{40}$ P. Metzner, L. Putzig, and I. Horenko, "Analysis of persistent nonstationary time series and applications," Communications in Applied Mathematics and Computational Science 7, 175-229 (2012).

${ }^{41}$ T. J. O'Kane, J. S. Risbey, C. Franzke, I. Horenko, and D. P. Monselesan, "Changes in the metastability of the midlatitude southern hemisphere circulation and the utility of nonstationary cluster analysis and split-flow blocking indices as diagnostic tools," Journal of the atmospheric sciences 70, 824-842 (2013).

${ }^{42}$ T. J. O’Kane, R. J. Matear, M. A. Chamberlain, J. S. Risbey, B. M. Sloyan, and I. Horenko, "Decadal variability in an ogcm southern ocean: Intrinsic modes, forced modes and metastable states," Ocean Modelling 69, 1-21 (2013).

${ }^{43}$ C. L. E. Franzke, T. J. O’Kane, D. P. Monselesan, J. S. Risbey, and I. Horenko, "Systematic attribution of observed southern hemisphere circulation trends to external forcing and internal variability," Nonlinear Processes in Geophysics 22, 513-525 (2015).

${ }^{44}$ J. S. Risbey, T. J. O’Kane, D. P. Monselesan, C. Franzke, and I. Horenko, "Metastability of northern hemisphere teleconnection modes," Journal of the Atmospheric Sciences 72, 35-54 (2015).

${ }^{45}$ T. J. O’Kane, J. S. Risbey, D. P. Monselesan, I. Horenko, and C. L. Franzke, "On the dynamics of persistent states and their secular trends in the waveguides of the southern hemisphere troposphere," Climate Dynamics 46, 3567-3597 (2016).

${ }^{46}$ T. J. O’Kane, D. P. Monselesan, J. S. Risbey, I. Horenko, and C. L. E. Franzke, “On memory, dimension, and atmospheric teleconnections," Mathematics of Climate and Weather Forecasting 3, 1-27 (2017).

${ }^{47}$ G. Nevo, N. Vercauteren, A. Kaiser, B. Dubrulle, and D. Faranda, "Statistical-mechanical approach to study the hydrodynamic stability of the stably stratified atmospheric boundary layer," Physical Review Fluids 2, 084603 (2017).

${ }^{48}$ J. S. Risbey, T. J. O’Kane, D. P. Monselesan, C. L. E. Franzke, and I. Horenko, “On the dynamics of austral heat waves," Journal of Geophysical Research: Atmospheres 123, 38-57 (2018).

${ }^{49}$ N. Vercauteren, V. Boyko, D. Faranda, and I. Stiperski, "Scale interactions and anisotropy in 
stable boundary layers," Quarterly Journal of the Royal Meteorological Society 145, 1799-1813 (2019).

${ }^{50}$ N. Vercauteren, V. Boyko, A. Kaiser, and D. Belušić, "Statistical investigation of flow structures in different regimes of the stable boundary layer," Boundary-Layer Meteorology 173, 143-164 (2019).

${ }^{51}$ V. Boyko and N. Vercauteren, "Multiscale shear forcing of turbulence in the nocturnal boundary layer: A statistical analysis," Boundary-Layer Meteorology , 1-30 (2020).

${ }^{52}$ E. Saggioro, J. de Wiljes, M. Kretschmer, and J. Runge, "Reconstructing regime-dependent causal relationships from observational time series," Chaos: An Interdisciplinary Journal of Nonlinear Science 30, 113115 (2020).

${ }^{53}$ A. J. Dowdy, A. Pepler, A. D. Luca, L. Cavicchia, G. Mills, J. P. Evans, S. Louis, K. L. McInnes, and K. Walsh, "Review of Australian east coast low pressure systems and associated extremes," Climate Dynamics 53, 4887-4910 (2019).

${ }^{54}$ S. Russo, J. Sillmann, and E. M. Fischer, "Top ten european heatwaves since 1950 and their occurrence in the coming decades," Environ. Res. Lett. 10, 124003 (2015).

${ }^{55}$ M. Li, Y. Yao, I. Simmonds, D. Luo, L. Zhong, and X. Chen, "Collaborative impact of the NAO and atmospheric blocking on European heatwaves, with a focus on the hot summer of 2018," Environ. Res. Lett. 15, 114003 (2015).

${ }^{56}$ L. Dieci and E. S. Van Vleck, "Computation of a few lyapunov exponents for continuous and discrete dynamical systems," Applied Numerical Mathematics 17, 275-291 (1995).

${ }^{57}$ W. K. Lau and K.-M. Kim, “The 2010 pakistan flood and russian heat wave: Teleconnection of hydrometeorological extremes," Journal of Hydrometeorology 13, 392-403 (2012).

${ }^{58}$ F. E. Otto, N. Massey, G. J. van Oldenborgh, R. G. Jones, and M. R. Allen, "Reconciling two approaches to attribution of the 2010 russian heat wave," Geophysical Research Letters 39 (2012).

${ }^{59}$ K. E. Trenberth and J. T. Fasullo, "Climate extremes and climate change: The russian heat wave and other climate extremes of 2010," Journal of Geophysical Research: Atmospheres 117 (2012).

${ }^{60}$ R. Dole, M. Hoerling, J. Perlwitz, J. Eischeid, P. Pegion, T. Zhang, X.-W. Quan, T. Xu, and D. Murray, "Was there a basis for anticipating the 2010 russian heat wave?" Geophysical Research Letters 38 (2011).

${ }^{61}$ S. Russo, A. Dosio, R. G. Graversen, J. Sillmann, H. Carrao, M. B. Dunbar, A. Singleton, P. Montagna, P. Barbola, and J. V. Vogt, "Magnitude of extreme heat waves in present climate 
and their projection in a warming world," Journal of Geophysical Research: Atmospheres 119, $12-500$ (2014).

${ }^{62}$ F. Pedregosa, G. Varoquaux, A. Gramfort, V. Michel, B. Thirion, O. Grisel, M. Blondel, P. Prettenhofer, R. Weiss, V. Dubourg, J. Vanderplas, A. Passos, D. Cournapeau, M. Brucher, M. Perrot, and E. Duchesnay, "Scikit-learn: Machine learning in Python," Journal of Machine Learning Research 12, 2825-2830 (2011).

${ }^{63}$ J. D. Hunter, "Matplotlib: A 2d graphics environment," Computing in Science \& Engineering 9, 90-95 (2007). 